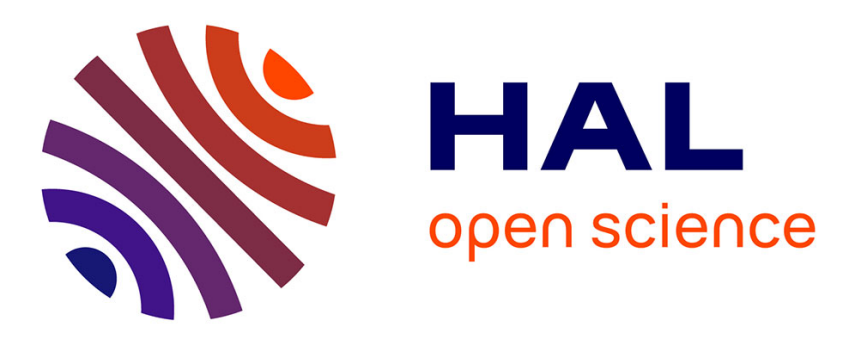

\title{
Shared Memory Parallelism for 3D Cartesian Discrete Ordinates Solver
}

Salli Moustafa, Ivan Dutka Malen, Laurent Plagne, Angélique Ponçot, Pierre Ramet

\section{- To cite this version:}

Salli Moustafa, Ivan Dutka Malen, Laurent Plagne, Angélique Ponçot, Pierre Ramet. Shared Memory Parallelism for 3D Cartesian Discrete Ordinates Solver. Joint International Conference on Supercomputing in Nuclear Applications and Monte Carlo 2013, Oct 2013, Paris, France. hal-00924989v2

\section{HAL Id: hal-00924989 \\ https://hal.inria.fr/hal-00924989v2}

Submitted on 5 May 2014

HAL is a multi-disciplinary open access archive for the deposit and dissemination of scientific research documents, whether they are published or not. The documents may come from teaching and research institutions in France or abroad, or from public or private research centers.
L'archive ouverte pluridisciplinaire HAL, est destinée au dépôt et à la diffusion de documents scientifiques de niveau recherche, publiés ou non, émanant des établissements d'enseignement et de recherche français ou étrangers, des laboratoires publics ou privés. 


\title{
Shared Memory Parallelism for 3D Cartesian Discrete Ordinates Solver
}

\author{
Salli Moustafa ${ }^{1}$, Ivan Dutka-Malen ${ }^{1}$, Laurent Plagne*1 $^{*}$, Angélique Ponçot ${ }^{1}$, and Pierre Ramet ${ }^{2}$ \\ ${ }^{1}$ EDF RED 1, Av du Général de Gaulle F92141 CLAMART CEDEX France \\ ${ }^{2}$ INRIA-University of Bordeaux, France
}

\begin{abstract}
This paper describes the performance of DOMINO, a 3D Cartesian SN Solver that implements two nested levels of parallelism (multicore+SIMD) on shared memory computation nodes. DOMINO solves a 3D full core PWR $k_{\text {eff }}$ problem involving 26 groups, 288 angular directions (S16), $46 \times 10^{6}$ spatial cells and $1 \times 10^{12}$ DoFs within 12 hours on a 32 core SMP node. This represents a sustained performance of 184 GFlops and $31 \%$ of the SMP node peak perfomance.
\end{abstract}

KEYWORDS: Deterministic transport, SN, multicore processor, wavefront, TBB, SIMD

\section{The DOMINO COCAGNE SN Solver}

COCAGNE is the name of the new nuclear core simulation system developed at EDF R\&D. Recently, a shared-memory parallel solver named DOMINO based on a discrete ordinates method has been introduced into the COCAGNE system.

\section{DOMINO Numerical Schemes}

DOMINO implements the Discrete Ordinates Method for the stationary neutron transport in a multi-dimensional Cartesian geometry. The quadrature used is the level symmetric one. In three-dimensions, this quadrature leads to $N(N+2)$ discrete directions where $N$ stands for the method's order. The space discretization corresponds to the Diamond Differencing scheme DD0. The DD0 element has 1 moment and 3 mesh-edge incoming fluxes per cell. The spatial problem is solved through the well-known sweep algorithm. We use a classical Diffusion Synthetic Acceleration (DSA) method.

\section{Shared Memory Parallel Implementation of DOMINO}

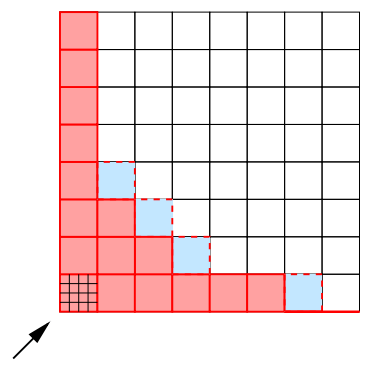

Figure 1: Wave front algorithm applied to the sweep across the mesh macrocells. The incoming fluxes come from the bottom left corner. The treated macrocells are pink colored while the white macrocells are still waiting. Only blue dashed border macrocells are ready for treatment and their respective indexes are stored in a task list from which a set of threads concurrently pick their work item. This list is dynamically updated each time a new macrocell has been treated.
The sweep operation is the most computationally intensive portion of DOMINO. For each incoming direction of the angular quadrature, the angular and volumic flux Degrees of Freedom (DoFs) of each spatial mesh cell must be updated. For simplicity's sake, we illustrate the parallel sweep on a 2D example with a DD0 spatial discretization scheme. Let $\left\{c_{i j}\right\}$ be the $n_{x} \times n_{y}$ cells of a 2D Cartesian spatial mesh. Let us consider $\Omega_{d}$ an angular direction incoming from the bottom left corner. Let $\left\{{ }^{d} \psi_{i j}^{x},{ }^{d} \psi_{i j}^{y}, \phi_{i j}\right\}$ be the corresponding incoming angular and volumic flux DoFs. In the sweep operation one has to treat each cell $c_{i j}$ by updating the volumic $\phi_{i j}$ and the angular outcoming ${ }^{d} \psi_{i+1 j}^{x}$ and ${ }^{d} \psi_{i j+1}^{y}$ DoFs that depend on the three ${ }^{d} \psi_{i j}^{x},{ }^{d} \psi_{i j}^{y}$ and $\phi_{i j}$ input values. This implies an ordering constraint for the sweep operation: a cell $c_{i j}$ can only be treated if the following two conditions are fulfilled:

$$
\begin{aligned}
& c_{i-1, j} \text { is already treated or } i=0, \\
& c_{i, j-1} \text { is already treated or } j=0 .
\end{aligned}
$$

Obviously $c_{00}$ is the first cell that can be treated but the second can be either $c_{10}$ or $c_{01} \ldots$ or both can be treated in parallel. We rely on the Intel TBB primitive parallel_do ${ }^{(\mathbf{1})}$ that enables a dynamic scheduling of the parallel tasks to implement the sweep. This parallel function allows a set of threads to execute the tasks from a task list which is dynamically updated. In the beginning, the task list contains the cell $c_{00}$. One of the running threads treats this cell and updates the task list to $\left\{c_{10}, c_{01}\right\}$ and so on. In order to reduce the overhead due to the thread scheduling, the cells are not treated individually but they are packed into groups of cells, called macrocells in the DOMINO implementation. All the cores of the supercomputing nodes are used in this parallel wavefront algorithm. Figure 1 illustrates this parallel sweep strategy.

During each sweep, the angular directions that belong to the same octant are computed via SIMD instructions allowing to perform simultaneously two double precision or four simple precision floating point operations.

In this paper the computations are made with one of the com- 
puting nodes of the EDF R\&D Ivanoé supercomputer ${ }^{1}$. This $1024 \mathrm{~GB}$ node is a 4 -socket Intel X7560 processor $(2.26 \mathrm{GHz}), 8$ cores per socket, and 4-way SIMD units totalizing 128 parallel FPUs that can be used concurrently. As a consequence, one can expect a 128 fold speed-up for an ideal parallel implementation of DOMINO compared to a sequential non-SIMD run time.

\section{Scalability and Efficiency}

We analyze the performance of the sweep operation for a simple 2-group $k_{\text {eff }}$ computation with $480^{3}$ spatial cells with different angular quadrature orders. Figure 2 and Table 1 sumarize the performance of the 2-level parallel implementation (multicore+SIMD) of DOMINO. Since the arithmetic intensity of the computation increases with the direction number, the sweep performance improves with the angular quadrature order. S2 and S4 timings are exactly the same because we use SIMD units to treat 4 angular directions that belong to the same octant at the same time. GFlops value is estimated by dividing the floating point operation number by the completion time:

$$
\text { GFlops }=\frac{25 \times N_{\text {cells }} \times N_{\text {dir }}}{\text { Time in nanosecond }},
$$

where the factor 25 accounts for the $20 \mathrm{add} / \mathrm{mult}$ operations and 1 division per cell (we count 5 flops for the division).

In the $\mathrm{S} 16$ case $\left(N_{\mathrm{dir}}=288\right)$, the sweep performance reaches 216 GFlops which corresponds to $37 \%$ of the peak performance given by (Single Precision):

$$
\begin{aligned}
\text { Peak } & =\text { ncores } \times \text { frequency } \times \text { SIMD width } \times \text { SIMD units } \\
& =32 \times 2.26 \times 4 \times 2=578 \text { GFlops } .
\end{aligned}
$$

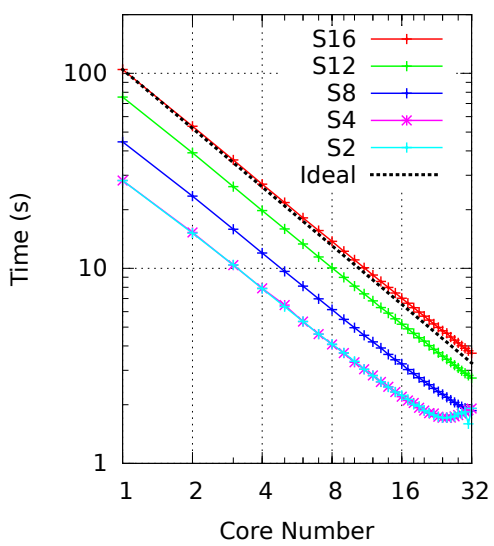

Figure 2: Time per sweep over all angular directions as a function of the core number (mesh: $480 \times 480 \times 480$ ).

\section{DOMINO/MCNP Comparison}

The benchmark used for calculations is described in reference. ${ }^{(2)}$ It corresponds to a simplified 3D PWR first core loaded

\begin{tabular}{c|c|c|c|c|c|c} 
Case & $N_{\text {dir }}$ & $\begin{array}{c}\text { Seq. } \\
\text { Time (s) }\end{array}$ & $\begin{array}{c}\text { 32-Core } \\
\text { Time (s) }\end{array}$ & $\begin{array}{c}\text { Speed } \\
\text { Up }\end{array}$ & GFlops & $\begin{array}{c}\% \text { Peak } \\
\text { Perf. }\end{array}$ \\
\hline S2 & $8 \times 1$ & 28.2 & 1.92 & 14.7 & 11.5 & $2 \%$ \\
\hline S4 & $8 \times 3$ & 28.2 & 1.92 & 14.7 & 34.7 & $6 \%$ \\
\hline S8 & $8 \times 10$ & 44.5 & 1.86 & 23.9 & 118.7 & $20.5 \%$ \\
\hline S12 & $8 \times 21$ & 75.6 & 2.74 & 27.6 & 169.6 & $29.3 \%$ \\
\hline S16 & $8 \times 36$ & 104.7 & 3.67 & 28.5 & 216.8 & $37.5 \%$
\end{tabular}

Table 1: Time per sweep over all angular directions as a function of the core number (mesh: $480 \times 480 \times 480$ ).

with 3 different types of fuel assemblies characterized by a specific Uranium-235 enrichment (low, medium and highly enriched uranium).

The calculation mesh is based on the cell pin mesh $(289 \times 289)$ and along the $\mathrm{z}$-axis, the height is subdived into 70 meshes for the 26-group calculation. For spatially converged results, meshes are refined by 2 in directions $\mathrm{x}, \mathrm{y}$ and $\mathrm{z}$. The angular discretization corresponds to a S16 level symmetric angular quadrature, which results in 288 directions in 3D. To simplify the comparisons to MCNP results, the 3D 26-group fluxes are integrated over energy and space. Practically, a group collapsing is performed from 26 to 2 energy groups with a boundary fixed at $0.625 \mathrm{eV}$, and a spatial integration over each pin-cell along the z-axis is made. Comparisons between DOMINO and MCNP presented in Table 2 are computed in the form:

\begin{tabular}{|c|c|c|c|c|c|}
\hline$k_{e f f}$ & $\begin{array}{l}\Delta k_{e f f} \\
(\mathrm{pcm})\end{array}$ & $\begin{array}{c}\left|\delta \phi_{1}\right| \\
(\%)\end{array}$ & $\begin{array}{c}\left|\delta \phi_{2}\right| \\
(\%)\end{array}$ & $\begin{array}{c}\left|\delta v \Sigma_{f} \phi\right| \\
(\%)\end{array}$ & $\begin{array}{l}\text { DOMINO } \\
\text { time }\end{array}$ \\
\hline 1.008361 & 12 & 0.69 & 0.80 & 0.34 & $12 \mathrm{~h} 06$ \\
\hline
\end{tabular}

$$
\begin{aligned}
& \Delta k_{e f f}=\frac{k_{e f f}^{D O M I N O}-k_{e f f}^{M C N P}}{k_{e f f}^{M C N P}} \times 10^{5} \text { in pcm, } \\
& \left|\delta \phi_{g}\right|=\max _{\left\{p_{i}\right\}}\left|\frac{\phi_{g}^{D O M I N O}\left(p_{i}\right)-\phi_{g}^{M C N P}\left(p_{i}\right)}{\phi_{g}^{M C N P}\left(p_{i}\right)}\right|,
\end{aligned}
$$

where $p_{i}$ stands for the pin cell index.

Table 2: Comparison to MCNP/DOMINO: S16, 26-group, $578 \times 578 \times 140$ spatial cells. $2.26 \mathrm{GHz}$ SMP node with 32 cores

This SN $k_{\text {eff }}$ calculation involved $1.05 \times 10^{12}$ DoFs obtained within 126 power iterations for a $10^{-5}$ stopping criterium. The DOMINO implementation reached 184.3 GFlops which corresponds to $31 \%$ of the SMP node peak performance.

Encouraged by these node performances, we are currently working on extending DOMINO to distributed memory machines.

\section{References}

1) A. Robison, M. Voss, and A. Kukanov, "Optimization via reflection on work stealing in TBB," Proc. Parallel and Distributed Processing, 2008. IPDPS 2008. IEEE International Symposium on, p. 1-8, IEEE, 2008.

2) T. Courau, L. Plagne, A. Ponçot, and G. Sjoden, "Hybrid Parallel Code Acceleration Methods in Full-Core Reactor Physics Calculations," Proc. Physor 2012 - Advances in Reactor Physics, 2010.

\footnotetext{
${ }^{1}$ Ranked 149 in Nov 2012 TOP 500 List
} 